\title{
CONGENITAL ARTERIOVENOUS FISTULA WITH AN ANEURYSM OF THE GREAT CEREBRAL VEIN AND HYDROCEPHALUS TREATED SURGICALLY
}

\author{
BY \\ JAMES B. GIBSON, ALEXANDER R. TAYLOR, and ALAN E. RICHARDSON \\ From the Departments of Pathology and Neurological Surgery, Royal Victoria Hospital, Belfast
}

Aneurysms occasionally develop from the great cerebral vein as a result of congenital arteriovenous fistulae. In a recent review Hirano and Terry (1958) referred to 18 cases, nine of which were confirmed at necropsy. The example of this rare condition reported here presented as a case of hydrocephalus showing certain noteworthy features. The condition responded well to surgical treatment, but the patient died after a fall. The connexions of the aneurysm were defined at necropsy.

\section{Case Report}

A baby girl of 6 months was brought for neurosurgical consultation because of obvious hydrocephalus in February, 1954. The pregnancy and delivery and the early development of the infant had been normal, and five weeks before she was examined she had been able to sit up almost unaided. At the time her balance had become impaired and she no longer raised her head spontaneously. The skull was seen to be expanded and superficial scalp veins were prominent. The skull circumference was $51.5 \mathrm{~cm}$.; the fontanelle was half closed but tense. Eye movements and pupillary reactions were normal; no other abnormalities were detected. In radiographs of the skull, opening of sutures and finger markings of the vault were seen but there was no evidence of the cause of the increased pressure. The transverse sinuses were in their normal position and the posterior fossa was not unduly shallow. At ventriculography, the intracranial pressure was high and an obstruction of the aqueduct was demonstrated, but not with the flaskshaped outline usually found in aqueduct stenosis. The obstruction (Fig. 1) began $2 \mathrm{~cm}$. below the third ventricle and increased caudally giving rise to a "rat tail" shadow. The aqueduct was displaced forwards and downwards suggesting a space-occupying lesion behind the pineal. To define this further, air was subsequently introduced by the lumbar route after the ventricular pressure had been lowered. The radiological appearances then were surprising (Fig. 2). All the subarachnoid cisterns were greatly dilated, particularly the cisterna ambiens. The sulci of the cerebral convexities were considerably enlarged up to the midline. These findings were interpreted to indicate defective absorption of fluid at the sagittal sinus as the primary cause of the hydrocephalus.
It was thought that the resultant rise in fluid pressure in the subarachnoid space had caused dilatation of the cisterns to such a degree that the cisterna ambiens had obstructed the aqueduct and so produced a type of hydrocephalus which was at least intermittently noncommunicating. To reverse this sequence an anastomosis between the spinal theca and the peritoneal cavity was made with polythene tubing on February 25, 1954. The immediate result was highly satisfactory: skull expansion ceased, the scalp veins became less distended, and normal development was resumed.

At the age of 1 year the child could stand with support the skull circumference had increased by only $2 \mathrm{~cm}$. At 18 months she could stand unaided; the fontanelles werea closed. She had a normal vocabulary and the mother thought her intelligence was normal. At 2 years she could walk, but her vocabulary seemed a little behind hero calendar age. At 3 years she appeared to be developingnormally. The skull circumference was $56.5 \mathrm{~cm}$. Thế

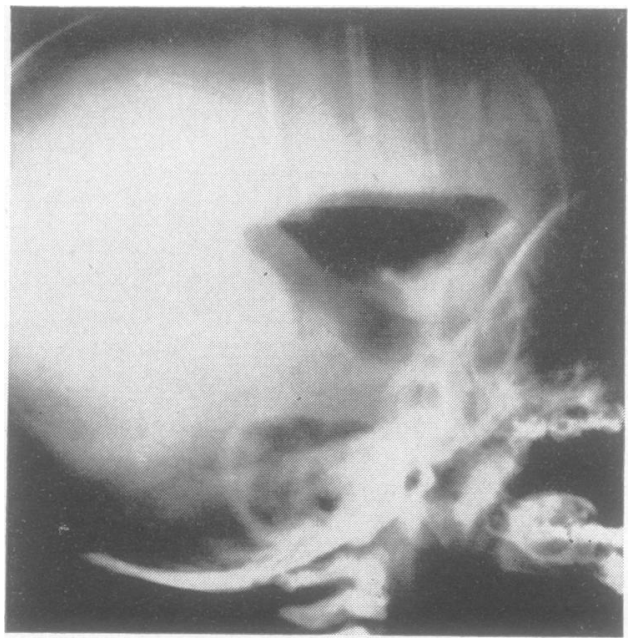

FIG. 1.-Ventriculography at 6 months of age. There is marked dilatation of the third ventricle and of the lateral ventricles (overlapping). The third ventricle is displaced upwards and forwards by the aneurysm. The aqueduct, initially dilated, tapers off distally. This unusual type of obstruction at the aqueduct has prevented air from entering the fourth ventricle. 


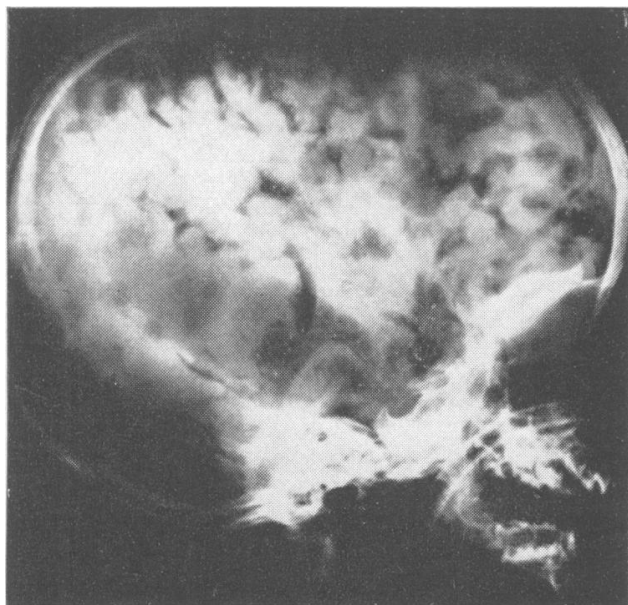

FIG. 2.-Encephalogram after introduction of air into lumbar theca at 6 months of age. Radiotranslucent patches mark dilated subarachnoid cisterns and enlarge sulci over the cerebral convexity. This unusual picture indicates a failure of absorption of cerebrospinal fluid.

scalp veins were still prominent but they were not distended; abduction of the left eye was slightly impaired. At $4 \frac{1}{2}$ years old she could walk and talk adequately. Her gait was a little unsteady at times but there were no localizing signs of cerebellar dysfunction.

At 4 years and 8 months, in April, 1958, she fell while playing, striking and bruising the left frontal area. She quickly became drowsy and was admitted to hospital with a left hemiparesis and bilateral extensor plantar responses. A diagnosis of acute intracranial bleeding was made but bilateral exploratory burr holes did not reveal any haematoma; the brain was not under tension. During the next two days she became more alert and recovered the power of the left limbs. Forty-eight hours after admission, however, she became cyanosed and apnoeic, apparently as a result of acute ischaemia of the brain-stem, and she died within two hours.

\section{Post-mortem Examination}

Permission was granted for examination of the head only. The operation sites in the skull were in a satisfactory state. The skull was deformed but not markedly enlarged. The brain weighed $2,000 \mathrm{~g}$. The convolutions were flattened and the brain tissue swollen. There was marked cerebral hydrocephalic deformity of the non-communicating type and the fourth ventricle was not enlarged; the lateral and medial apertures of the ventricle were patent. The subarachnoid cisterns were not enlarged. The brain was fixed entire together with the tentorium cerebelli, and subsequently sectioned in the coronal plane (Fig. 3). The ventricular system was obstructed at the aqueduct. The obstruction was caused by an external spherical swelling about $4 \mathrm{~cm}$. in diameter with a fibrous wall. The swelling was an aneurysm extending downwards and forwards from the anterior end of the incisura tentorii and separating the brain-stem from the splenium. The anterosuperior surface of this aneurysmal sac had stretched the posterior wall of the third ventricle, obliterated the recesses, and become firmly adherent to the pineal which was flattened antero-posteriorly. Hydrocephalic dilatation of the lateral ventricles and the upward and lateral pressure of the swelling into the calcarine fissures had thinned and flattened the medial surfaces of the occipital lobes so that the medial walls of the posterior horns of the lateral ventricles were stretched and attenuated. The usual deformities of cerebral hydrocephalus due to obstruction of the aqueduct were present, the cortex and white matter being reduced to a thickness of about $1.5 \mathrm{~cm}$. in most places. The choroid plexuses were normal. The aneurysmal mass stretched the quadrigeminal plate below it to a thin membrane and the aqueduct was reduced to a transverse slit (Fig. 3). The superior vermis was compressed and formed the floor of a concavity which included also the medial parts of the lateral lobes of the cerebellum on either side. The fourth ventricle was slightly reduced in size.

There were no scars of the tentorium cerebelli or anything else to suggest previous tears and there was no evidence of haemorrhage from the aneurysm at any time. The aneurysm involved the posterior portion of the great cerebral vein and bulged downwards from the origin of the straight sinus at its origin at the apex of the tentorium. The straight

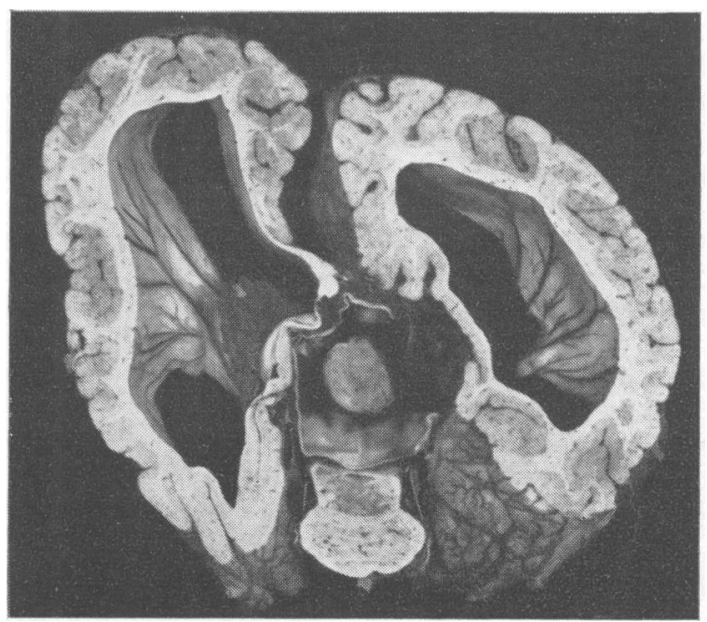

FIG. 3.-Coronal section through the aneurysm and the hydrocephalic brain at the level of the splenium, including the pons. A pale ball thrombus occupies part of the aneurysm. The aqueduct is patent but compressed below the aneurysm. The cerebral veins 


\section{JAMES B. GIBSON, ALEXANDER R. TAYLOR, AND ALAN E. RICHARDSON}

sinus was dilated to $1 \cdot 3 \mathrm{~cm}$. diameter and had a thick leathery wall. The torcula was an irregularly shaped cavity about $2.5 \mathrm{~cm}$. wide. The right transverse sinus was also dilated $(1 \cdot 2 \mathrm{~cm}$. wide) and thick walled. The left transverse sinus narrowed along its course and was $3 \mathrm{~mm}$. wide at the point where the tentorium had been cut anteriorly. The superior sagittal sinus was $4 \mathrm{~mm}$. wide. The aneurysm contained a white ball thrombus loosely adherent to its wall and not organized. The wall of the sac was about $1 \mathrm{~mm}$. thick and microscopical examination showed that it was composed largely of elastic fibres and hyaline fibrous tissue formed in the intima of the vein. A similar intimal proliferation caused the thickening of the walls of the neighbouring venous sinuses.

On account of the stretching of the region, the vascular connexions of the aneurysm were identified with some difficulty and only after microscopical examination. It was possible, however, to locate and recognize the major veins and arteries and these are shown in Fig. 4. Angiomas were not found. The vessels were identified as follows.

Anteriorly both superficial middle cerebral veins were dilated and their entries into the cavernous sinuses were enlarged. Over the lateral surfaces of the hemispheres, the right vein was more dilated than the left. The right deep middle cerebral vein consisted of several small dilated vessels which drained into the basal vein. The right basal vein pursued an approximately normal course lateral to the cerebral peduncle and drained into the great cerebral vein where it was dilated into the aneurysm (Fig. 4). The left deep middle cerebral vein was dilated near its termination in the left basal vein on the cerebral peduncle. This left basal vein ended abnormally on the medial surface of the hemisphere behind the thalamus, external to the choroid plexus, and, together with the thalamo-striate and choroidal veins, gave origin to the left internal cerebral vein. The right internal cerebral vein was also identified by its origin in the thalamo-striate and choroidal veins on that side. Two-thirds of the way along its course it received an anomalous arterial tributary from the posterior cerebral artery, an arterio-venous fistula. The terminal third of the vein was dilated and varicose down to its termination in the great cerebral vein. The proximal $1 \mathrm{~cm}$. of the great cerebral vein, between its origin in the union of the two internal cerebral veins and the aneurysm, was also dilated but not aneurysmal. On the right side it received a single small tributary from the posterior wall of the third ventricle. At the point of origin of the aneurysm the vein was joined by an arteriovenous fistula which was $2 \mathrm{~mm}$. wide, a long arterial vessel which ran across the

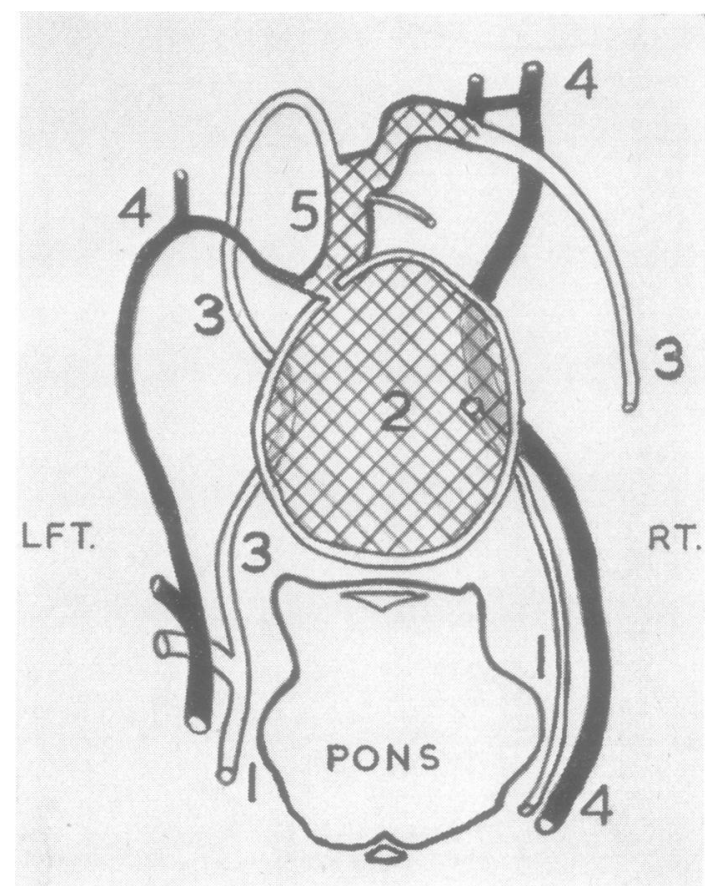

FIG. 4.-Semidiagrammatic representation of the aneurysm (2) at the distal end of the great cerebral vein and its vascular connexions. Branches of the posterior cerebral artery (4) are shown $?$ in black. The artery on the right lies in front of the aneurysm. There are arteriovenous fistulae, on the left to the dilated anterior portion of the great cerebral vein (5) where it enters the aneurysmal portion, and on the right to the terminal portion of the $\bar{c}$. internal cerebral vein (3). Vessels containing mixed blood are $\bar{\tau}$ cross-shaded. The right basal vein (1) ends in the aneurysm.? The left basal vein (1) terminates abnormally at the origin of the left internal cerebral vein (3) which then passes in front of the aneurysm.

medial surface of the occipital lobe from the left posterior cerebral artery. The conjoined vessels entered the aneurysm at an angle, forming an opening $3 \mathrm{~mm}$. wide in the lateral wall of the aneurysm. There were two other openings leading into the aneurysm, one of which was formed by the right basal vein and was $2 \mathrm{~mm}$. wide. The inferior sagittal sinus, which was dilated, opened into the upper surface of the aneurysm close to the termination of the latter in the straight sinus. On microscopical examination, veins over the cerebrum, in the basal nuclei, and over the cervical portion of the spinal cord were seen to be dilated and their walls were fibrous. The arteries of the circle of Willis were normal.

\section{Discussion}

Death resulted from the trauma sustained by the child while playing. Post-traumatic cerebral oedema was probably enhanced by the effects of the ball 
thrombus in the aneurysm. Increased secretory activity on the part of the choroid plexus may have contributed to upset the delicate balance between production and absorption of cerebrospinal fluid which had been established by the previous thecoperitoneal drainage.

In the cases of Lumsden (1947) and of Clément, Gerbeaux, Combes-Hamelle, Pertuiset, and Pétranca, (1954, Case 1) the aneurysms were found in infants of 7 months and 1 month respectively and had caused subarachnoid haemorrhages. The ages of these cases and the location of the lesion might suggest that aneurysms of this type are caused by trauma at birth. In our case, however, there was nothing to indicate any previous tear of the tentorium and the existence of arteriovenous fistulae permits elimination of trauma as an aetiological factor with even greater certainty. Hirano and Terry (1958) have listed the arterial connexions of the aneurysms in the 18 cases in the literature. In 10 instances the posterior cerebral arteries were involved alone, as in our case, or in combination with other cerebral arteries. The anterior cerebral and pericallosal arteries were involved in other instances. Russell and Nevin (1940) reported the absence in their first case of any venous channels leading through the jugular foramina, and the terminations of the superficial middle cerebral veins in the cavernous sinuses were dilated. These terminations were dilated in our case too, but unfortunately the jugular foramina were not examined; the dilatation of the right transverse venous sinus suggests that drainage of blood was normal on that side, but in view of the narrowing of the other transverse sinus, the connexion of the left sigmoid sinus with the jugular vein may have been absent. Many of the deep and superficial cerebral veins on both sides were dilated and tortuous because of the increased entry of blood from the fistulae, but it was possible to identify all the major channels with normal anatomical elements. Thus, there was no genuinely angiomatous element in the anomaly and this seems to have been true also of the other cases which are discussed here, The extent of the venous anomalies described has varied considerably and may depend in part on the size of the arteriovenous fistulae. In the case of Jaeger and Forbes (1946), in which both arteries and veins were considerably enlarged, the fistula was big enough to lead to cardiac enlargement. The fistulae and the resultant aneurysm constitute a single entity, but it is to be expected that the lesion might occur from time to time in combination with arteriovenous malformations in other parts of the brain.

Superficial scalp veins are often dilated and prominent as in our case, because of their connexions with the cerebral venous system. Dilatation of superficial veins may be the presenting symptom (Boldrey and Miller, 1949). In the case of Hirano and Terry (1958) dilatation of venous channels caused protuberance of the right eye.

At necropsy, the walls of many of the cerebral veins were thick and fibrous, in keeping with the considerable degree of venous hypertension which certainly prevailed during life. The hypertension was probably the main factor impeding resorption of cerebrospinal fluid through the arachnoid granulations. In cases where subarachnoid haemorrhages have occurred, thickening of the leptomeninges may also interfere with resorption (Askenasy, Herzberger, and Wijsenbeek, 1953) but this complication was not present in our case. The dilatation of the subarachnoid cisterns noted before operation indicated that absorption of cerebrospinal fluid had been considerably impaired and this element of the hydrocephalus was relieved by thecal drainage. After operation the circumference of the head and the child's development began to approach normal. The cisterns were not dilated at death. The residual clinical signs were probably caused directly by the mass of the aneurysm. Possibly complete recession of the hydrocephalus was impossible at this time. In the case of Hirano and Terry (1958) the arterial feeders of the aneurysm closed spontaneously and completely but the hydrocephalus did not recede. Askenasy et al. (1953) suggest that deviation of arterial blood from the brain through the fistulae may increase the degree of cerebral atrophy caused by the elevation in cerebrospinal fluid pressure, but the clinical observations in our case do not support this suggestion. Although failure of absorption seems to have played the chief part in the genesis of the hydrocephalus, the direct effects of the aneurysm in compressing the aqueduct cannot be neglected, and the hydrocephalus may have been maintained to some extent by this means. The aqueduct was not completely closed, but obstruction may have been intermittent, as apparently in the case of Jaeger and Forbes (1946). The balance between production and absorption of cerebrospinal fluid achieved by the operation was a delicate one and was eventually upset by trauma.

Hydrocephalus was absent in a number of the cases reported in adults and older children (Oscherwitz and Davidoff, 1947; French and Peyton, 1954) but it has been the main symptom in children. The experience in the present case suggests that cerebrospinal fluid drainage operations have a useful part to play in its relief. Most often surgical treatment of the condition has taken the form of clipping the arterial feeders as fully as possible. Hirano and Terry (1958) point out that the chief value of such 
operations probably lies in preventing subarachnoid haemorrhage and this complication has occurred often enough to deserve serious consideration. Personality changes have been recorded by French and Peyton (1954).

The presence of dilated superficial veins on the scalp in cases of hydrocephalus should arouse suspicion of the existence of an aneurysm of the great cerebral vein, and occasionally eye signs may suggest the presence of a lesion in the region of the pineal. In the present case there was slight abduction weakness of the left eye. Wolfe and France (1949) mention papilloedema and also evidence of defective innervation of the intrinsic and extrinsic muscles of the left eye. The patient of Jaeger and Forbes developed a squint while under observation. Eye signs were absent in the cases of Alpers and Forster (1945) and of Oscherwitz and Davidoff (1947). The diagnosis has most often been made by angiography but other radiological methods may be of use. The peculiar outline of the upper end of the aqueduct in the ventriculograms in our case may be paralleled in others. In several cases in adults the wall of the aneurysm has been partially calcified so that the dilated vessel was outlined (Alpers and Forster, 1945; Oscherwitz and Davidoff, 1947; Boldrey and Miller, 1949; Wolfe and France, 1949). The dilatation of the transverse sinuses might also in its turn lead to increased skull markings over the vessels, but the location of the sinuses on the skull is normal and they are not maintained in the foetal position as in congenital hydrocephalus due to atresia of the foramen of Magendie (Taggart and Walker, 1942; Gibson, 1955).

\section{Summary}

In a case of hydrocephalus caused by arteriovenous fistulae and an aneurysm of the posterior part of the great cerebral vein, the history and the necropsy findings in the head are recorded. Hydrocephalus and dilatation of superficial scalp veins were noted at 6 months of age. The hydrocephalus was found to be due in part to a failure of absorption of cerebrospinal fluid which probably resulted from high venous pressure in the superior sagittal sinus. A surgical spinal theco-peritoneal anastomosis was performed with good results. Since the operation compensated for the failure of absorption of cerebrospinal fluid by the natural routes, defective absorption appears to have played an important part in causing the hydrocephalus. The child died as the result of an accident when 4 years 8 months old. The aneurysm was found to have caused persistent but incomplete obstruction of the aqueduct. Associated vascular anomalies were marked dilatation of the anterior part of the great cerebral vein and of a portion of the right internal cerebral vein, arteriovenous fistulae on each side arising from branches of the posterior cerebral arteries, anomalous termination of the left basal vein, and elongation and dilatation of veins in many parts of the central nervous system. Angiomatous formations were not present. The case is discussed in relation to those reported by others.

We wish to thank Professor J. H. Biggart for advice and encouragement and Mr. D. McA. Mehaffey for the photographs.

\section{REFERENCES}

Alpers, B. J., and Forster, F. M. (1945). Arch. Neurol Psychiat (Chicago), 54, 181.

Askenasy, H. M., Herzberger, E. E., and Wijsenbeek, H. S. (1953). Neurology, 3, 213. Boldrey, E., and Miller, E. R. (1949). Arch. Neurol. Psychiat.

Clément, R., Gerbeaux, J., Combes-Hamelle, A., Pertuiset, B., and Pétranca, C. (1954). Presse méd., 62, 658.

French, L. A., and Peyton, W. T. (1954). J. Neurosurg., 11, 488.

Gibson, J. B. (1955). J. Neuropath. exp. Neurol., 14, 244.

Girano, A., and Terry, R. D. (1958). Ibid., 17, 424.

Jaeger, R., and Forbes, R. P. (1946). Arch. Neurol. Psychiat. (Chicago), 55, 591 .

Lumsden, C. E. (1947). J. Path. Bact., 59, 328.

Oscherwitz, D., and Davidoff, L. M. (1947). J. Neurosurg. 4, 539.

Russell, D. S., and Nevin, S. (1940). J. Path. Bact. 51, 375.

Taggart, J. K., and Walker, A. E. (1942). Arch. Neurol. Psychiat. (Chicago), 48, 583 .

Wolfe, H. R. I., and France, N. E. (1949). Brit. J. Surg., 37, 76. 